\title{
The Efficacy and Safety of Laser Lithotripsy in Pregnancy
}

\author{
Amir Reza Abedi ${ }^{1}$, Farzad Allameh ${ }^{2 *}$, Mohammad Reza Razzaghi ${ }^{3}$, Behrouz Fadavi ${ }^{1}$, Hamidreza Qashqai ${ }^{1}$, \\ Saman Najafi ${ }^{3}$, Arash Ranjbar ${ }^{1}$, Manouchehr Bashirian ${ }^{1}$ \\ ${ }^{1}$ Department of Urology, Shohada-e-Tajrish Hospital, Shahid Beheshti University of Medical Sciences, Tehran, Iran \\ ${ }^{2}$ Center of Excellence for Training Laser Application in Medicine, Shohada-e- Tajrish Hospital, Ministry of Health, Tehran, \\ Iran \\ ${ }^{3}$ Laser Application in Medical Sciences Research Center, Shahid Beheshti University of Medical Sciences, Tehran, Iran
}

\section{*Correspondence to \\ Farzad Allameh, MD-MPH; Laser Application in Medical Sciences Research Center, Shahid Beheshti University of Medical Sciences, Tehran, Iran. \\ Tel: +98-21 22718021 \\ Fax: +98-2122749221; \\ Email: farzadallame@gmail.com}

Published online 28 March 2017

\begin{abstract}
Introduction: The aim of our study was to assess the efficacy and safety of laser lithotripsy in pregnant patients.

Methods: In this retrospective study, we reviewed the 15 pregnant women who have been treated for ureteral stones with semi-rigid ureteroscope and holmium laser at our center between Januarys 2007and April 2015.

Results: The mean age of patients and mean gestational age was 29.3 years old 27.3 weeks respectively. Mean size of stones was $7.84 \mathrm{~mm}$. Twelve patients had renal colic, and hematuria was found in 3 cases. Irritative urinary symptoms such as frequency and urgency detected in 6 ones and 2 patients had fever. The stone of all patients were fragmented by using holmium laser lithotripter. In 5 patients stone residual fragments were removed by grasper while other 10 patients were left to pass fragments spontaneously. No intraoperative and postoperative urological or obstetric complication was seen.

Conclusion: Laser lithotripsy is safe and efficacious in pregnant patients who have ureteral stone that does not respond to conservative management.

Keywords: Complication; Laser lithotripsy; Pregnancy; Ureter stone; Urolithiasis.
\end{abstract}

\section{Introduction}

Renal colic is the most common non-obstetrical cause of hospitalization during pregnancy. ${ }^{1}$ Urolithiasis may cause urinary stasis in pregnant cases that can lead to urinary tract infection and pyelonephritis which are associated with obstetric complications such as preterm delivery and spontaneous abortion. ${ }^{2}$

Between $80 \%$ to $90 \%$ of urinary stones during pregnancy are diagnosed in the second or third trimesters. ${ }^{3}$ Fifty to $80 \%$ of stones during pregnancy tend to pass spontaneously with conservative treatment, ${ }^{4}$ but patients whose renal colic did not resolve despite conservative treatment during follow-up period underwent ureterorenoscopy (URS). ${ }^{5}$ The main benefit of URS is visualization of the ureter and renal pelvis that enables detection and treatment of ureteral stones. ${ }^{6}$ All types of stones can be fragmented with a certain degree of safety with holmium: yttrium-aluminium-garnet (YAG) laser lithotripsy. ${ }^{7}$

\section{Methods}

In this retrospective study, we reviewed 45 pregnant women afflicted with ureteral stones who underwent URS and holmium: YAG laser at our center between January 2007 and June 2016. Hospital charts were reviewed; our data include patient's age, presenting symptoms, diagnostic methods, past history of urolithiasis or urological interventions, localization and size of stone, and stage of pregnancy.

Laboratory data including complete blood count (CBC), urea and creatinine serum levels, as well as urine and blood cultures (if sepsis was suspected) were recorded. The diagnosis of ureteral stone during pregnancy was based on the clinical manifestations of the patients, presence of microscopic hematuria in urinalysis, and trans-abdominal ultrasound findings. The stones below the iliac artery pulsation in URS were accepted as lower ureteral stones and the ones above this pulsation were classified as upper ureteral stones.

Conservative treatment including intravenous fluid replacement and safe analgesics was started on all hospitalized patients. All patients underwent preoperative intravenous antibiotic prophylaxis and our protocol was one dose of second or third generation cephalosporin intravenously 1 hour before the URS. URS procedures 
were delayed in patients with active urinary system infection until their urine cultures became sterile, except in patients with the doubt of pyonephrosis.

URS procedures were done using a $9.5 \mathrm{~F}$ (Karl Storz Inc., Germany) semi-rigid ureteroscope under direct endoscopic vision without fluoroscopic guidance. Ureters were accessed using 0.035 inch guide wires. The stones were fragmented using holmium-YAG laser (Iranian National Laser Center, Iran).

If there were ureteral edema, presence of a concomitant renal stone, significant residual stones or ureteral trauma caused by URS, double J stent (RÜSCH-Teleflex Inc., USA) was inserted. Double J stent was removed 2 weeks after procedure.

During the early postoperative period, obstetric care was offered to all patients to avoid medico-legal issues and to attain maternal and fetal well-being.

\section{Results}

The mean age of patients and mean gestational age was 29.3 years old and 27.3 weeks (13-31 weeks) respectively. The mean size of stones was $7.84 \mathrm{~mm}$ (5-9 mm). Patients' characteristics were shown in Table 1. Eighteen patients had history of urolithiasis, 6 had history of extracorporeal shockwave lithotripsy (ESWL), 15 had history of transurethral lithotripsy and 3 had history of spontaneous stone passage.

The presenting symptoms were renal colic in 36 patients, hematuria in 9, irritative symptoms such as urinary frequency and urgency in 18 and fever in 6 patients. Twenty-eight patients had right and the other 17 had left ureteral stones. Stones were detected in distal part of left and right ureter and proximal part of right ureter in 17 , 16 , and 5 cases, respectively. Lithotripsy time duration was $12.6 \pm 2.3$. The stones of all patients were fragmented using laser lithotripter. Stone fragments of 15 patients

Table 1. Patient Characteristics

\begin{tabular}{ll}
\hline Patient (n) & 45 \\
\hline Age (y) & 29.3 \\
Mean of gestational age (wk) & 27.3 \\
\hline First trimester (No. of patients) & 9 \\
\hline Second trimester (No. of patients) & 24 \\
Third trimester (No. of patients) & 12 \\
Laterality & 17 \\
\hline Left & 28 \\
Right & 18 \\
History of urolithiasis & \\
Symptoms & 36 \\
Renal colic & 3 \\
Fever & 9 \\
Hematuria or pyuria & 18 \\
Irritative symptoms & 18 \\
Stone diagnosed by ultrasound & $7.84 \mathrm{~mm}$ \\
Mean of stone size on ultrasound (mm) & 6 \\
\hline Positive urine culture & \\
\hline
\end{tabular}

were removed by grasper while other 30 patients were left to pass fragments spontaneously. Only 3 patients had residual ureteral stones after 3 weeks. Stone free rate was $93.3 \%$. Six patients had positive urine cultures and one of them had pyonephrosis which was managed by nephrostomy insertion and delayed URS.

Intraureteral JJ stent was inserted in 30 patients because of edema caused by impacted stones, intraurethral trauma during URS or concomitant renal stone. Double J stent was removed 2-3 weeks later in 21 patients but JJ stents of 3 patients with concomitant renal stones was removed after successful ESWL performed during postnatal period. In these 3 patients we changed the stent every 6 weeks to restrain encrustation. Detail of procedure and outcome was outlined in Table 2.

No intraoperative urological or obstetric complication was seen. During postoperative hospitalization preterm contraction was seen in 2 patients who were successfully treated by tocolytics. Urinary tract infection occurred in 2 patients who were treated by a safe group of antibiotics. Pain completely disappeared in 36 cases postoperatively; but mild pain remained in 4 patients who were treated with acetaminophen. Obstetrics consultations were done in all cases before and after procedure. All patients had normal delivery and their babies had good health.

\section{Discussion}

Hydronephrosis is reported in $90 \%$ of pregnant women in their third trimesters, and it may persist up to 12 weeks postpartum. It is more common on the right side. ${ }^{8}$ Urolithiasis can lead to hydronephrosis and eventually pyelonephritis that can result in obstetric complications such as preterm labor and low birth weight new-born. ${ }^{2}$

It was estimated that there is one case of urolithiasis requiring active treatment in every 1500 pregnancies. ${ }^{9}$ Fifty to $80 \%$ of stones during pregnancy pass spontaneously with conservative treatment ${ }^{4}$ but, $20 \%$ to $30 \%$ of cases will need some form of active treatment such as placing a percutaneous nephrostomy (PCN) tube or performing ureteroscopic procedures. ${ }^{10}$

The purpose of the treatment is to diminish maternal pain, to prevent renal dysfunction and urinary tract infection and to decrease obstetric complications. ${ }^{2}$ ESWL is contraindicated during pregnancy because of its

Table 2. Details of Procedure and Outcomes

\begin{tabular}{|c|c|}
\hline Stone Location & \\
\hline Proximal & 5 \\
\hline Distal & 40 \\
\hline Double-J stent insertion & 30 \\
\hline \multicolumn{2}{|l|}{ Type of lithotripter } \\
\hline Holmium-YAG laser lithotripsy & 45 \\
\hline \multicolumn{2}{|l|}{ Postoperative complication } \\
\hline Preterm uterine contraction & 2 \\
\hline Urinary tract infection & 2 \\
\hline Duration of lithotripsy (min) & $12.6 \pm 2.3$ \\
\hline
\end{tabular}


adverse effect upon the fetus. ${ }^{11}$ The development of the semi-rigid or flexible ureteroscope is one of the reasons why URS procedures are becoming more common during pregnancy with high efficacy. Ulvik et al showed that the ureteroscopic procedure has not been associated with adverse effects in 24 pregnant cases. ${ }^{12}$ Similar results were reported by Lifshitz and Lingeman ${ }^{13}$ and Watterson et al. ${ }^{14}$ Lemos et a ${ }^{15}$ reported no obstetrical complications after URS for diagnosis and/or treatment. In our study, we did not see any ureteral perforation or obstetric complications. During pregnancy, urolithiasis can be managed by ureteral stenting or insertion of a percutaneous nephrostomy (PCN) tube. However, some risks such as urinary tract infection, and encrustation related to PCN tube and ureteral stents have been reported. ${ }^{16,17}$ This encrustation can require frequent stent changes, which can be every 6-8 weeks. These procedures can cause complications such as UTI, which can be associated with obstetric complications. Placing nephrostomy tube in pregnancy has similar complications. ${ }^{12,16,17}$ In the study of Parulkar et $\mathrm{al}^{4}$ on 70 pregnant women with urinary stones, 19 of 70 patients required invasive treatment. One-third of patients needed subsequent interventions because of encrustation, severe irritative symptoms or migration. Denstedt and Razvi ${ }^{18}$ reported that ureteral stent placing is not recommended before 22 weeks of gestation, as a substitute of ureteral stent, percutaneous nephrostomy tube is recommended.

Another option is URS and stone fragmentation that can be done by some lithotripters. ${ }^{6,15}$ Bozkurt et al ${ }^{19}$ used holmium laser with success in 17 (53.1\%) patients and pneumatic lithotripter in $8(25 \%)$ patients. They found that the pneumatic lithoclast and holmium: YAG laser to be safe.

The advantages of holmium laser lithotripters can be enumerated including the holmium: YAG laser can be used through a rigid or flexible ureteroscope ${ }^{20}$ all types of stones with varying compositions can be fragmented into relatively small particles which can be easily eliminated. ${ }^{21}$ Holmium laser lithotripters compared with pneumatic lithotripters, produce weak shock waves with minimal tissue penetration, thus theoretically limiting the risk of fetal injury. The probability of stone migration is less when Holmium laser lithotripter was used. ${ }^{20,21}$

Laing et $\mathrm{al}^{22}$ reported a series review consisting of 116 pregnant women who underwent URS for ureteral stone treatment: holmium laser was used with success in 27 (23\%) patients and a pneumatic lithotripter in $21(18 \%)$ patients.

This study had some limitations. Firstly, our study like other studies was a retrospective case series without a control arm, and this inevitably introduces selection bias. The second limitation was related to the small sample size, and finally we were not able to stratify our patients by the gestational age and analyze the outcomes in subgroups.

\section{Conclusion}

It seems that laser lithotripsy is safe and efficacious in pregnant patients who have ureteral stone that does not respond to conservative management. Further prospective and randomized studies should be done to approve this result.

\section{Ethical Considerations}

Our study was a retrospective review and have been approved by ethical committee of the Institutional Review Board of the Shahid Beheshti University of Medical Sciences.

\section{Conflict of Interests}

The authors do not have any proprietary interests in this study.

\section{References}

1. Rodriguez PN, Klein AS. Management of urolithiasis during pregnancy. Surg Gynecol Obstet. 1988;166:103-106.

2. Negru I, Pricop C, Costachescu G. Renal colic in pregnancy (Romanian). Rev Med Chir Soc Med Nat Iasi. 2010; 114(2):439-444.

3. Swanson SK, Heilman RL, Eversman WG. Urinary tract stones in pregnancy. Surg Clin N Am. 1995;75:123-142.

4. Parulkar BG, Hopkins TB, Wollin MR, Howard PJ Jr, Lal A. Renal colic during pregnancy: a case for conservative treatment. J Urol. 1998;159(2):365-368.

5. Semins MJ, Trock BJ, Matlaga BR. The safety of ureteroscopy during pregnancy: a systematic review and meta-analysis. J Urol. 2009;181(1):139-143. doi:10.1016/j.juro.2008.09.029.

6. Scarpa RM, De Lisa A, Usai E. Diagnosis and treatment of ureteral calculi during pregnancy with rigid ureteroscopes. J Urol. 1996;155:875-877.

7. Sofer M, Watterson JD, Wollin TA, Nott L, Razvi H, Denstedt JD. Holmium:YAG laser lithotripsy for upper urinary tract calculi in 598 patients. J Urol. 2002;167:31-34.

8. Boridy IC, Maklad N, Sandler CM. Suspected urolithiasis in pregnant women: imaging algorithm and literature review. AJR Am J Roentgenol. 1996;167:869-875.

9. Kroovand L. Stones in pregnancy and in children. J Urol. 1992;148(3 Pt 2):1076-1078.

10. Biyani CS, Joyce AD. Urolithiasis in pregnancy II: management. BJU Int. 2002;89:819-823.

11. Ohmori K, Matsuda T, Horii Y, Yoshida O. Effects of shock waves on the mouse fetus. J Urol. 1994;151(1):255-258.

12. Ulvik NM, Bakke A, Høisaeter PA. Ureteroscopy in pregnancy. J Urol. 1995;154(5):1660-3.

13. Lifshitz DA, Lingeman JE. Ureteroscopy as a first-line intervention for ureteral calculi in pregnancy. J Endourol. 2002;16(1):19-22.

14. Watterson JD, Girvan AR, Beiko DT, et al. Ureteroscopy and holmium:YAG laser lithotripsy: an emerging definitive management strategy for symptomatic ureteral calculi in pregnancy. Urology. 2002;60:383-387.

15. Lemos GC, El Hayek OR, Apezzato M. Rigid ureteroscopy for diagnosis and treatment of ureteral calculi during pregnancy. Int Braz J Urol. 2002;28(4):311-315.

16. Rana AM, Aquil S, Khawaja AM. Semirigid ureteroscopy and pneumatic lithotripsy as deWnitive management of obstructive ureteral calculi during pregnancy. Urology. 2009;73:964-7.

17. Kavoussi LR, Albala DM, Basler JW, Apte S, Clayman RV. Percutaneous management of urolithiasis during 
pregnancy. J Urol. 1992;148:1069-1071.

18. Denstedt JD, Razvi H. Management of urinary calculi during pregnancy. J Urol. 1992; 148:1072-1074.

19. Bozkurt Y, Penbegul N, Soylemez H, et al. The efficacy and safety of ureteroscopy for ureteral calculi in pregnancy: our experience in 32 patients. Urol Res. 2012;40(5):531-5. doi: 10.1007/s00240-011-0454-y.

20. Geavlete P, Mulåescu R, Georgescu D. Flexible ureteroscopic approach in upper urinary tract pathology.
Chirurgia (Bucur). 2006;101(5):497-503

21. Teichman JM, Vassar GJ, Bishoff JT, Bellman GC. Holmium:YAG lithotripsy yields smaller fragments than lithoclast, pulsed dye laser or electrohydraulic lithotripsy. J Urol. 1998;159:17-23

22. Laing KA, Lam TB, McClinton S, Cohen NP, Traxer O, Somani BK. Outcomes of ureteroscopy for stone disease in pregnancy: results from a systematic review of the literature. Urol Int. 2012;89:380-386. 